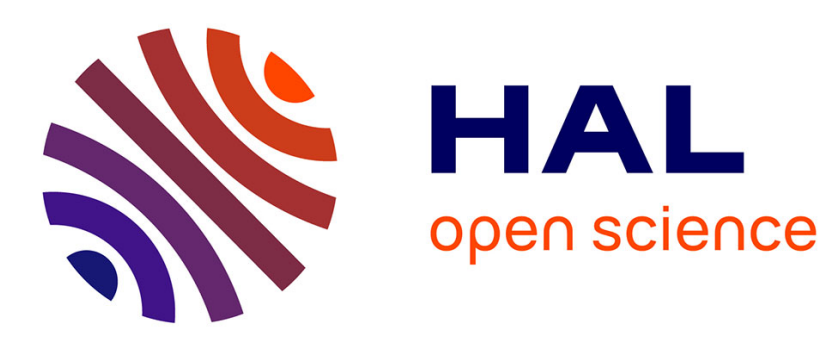

\title{
Numerical Modelling of Turbulent Two Phase Flows Using the Two Fluid Approach
}

\author{
Jean-Marc Hérard
}

\section{To cite this version:}

Jean-Marc Hérard. Numerical Modelling of Turbulent Two Phase Flows Using the Two Fluid Approach. 17th AIAA Computational Fluid Dynamics Conference, Jun 2005, Toronto, Canada. 10.2514/6.2003-4113 . hal-01583852

\section{HAL Id: hal-01583852 \\ https://hal.science/hal-01583852}

Submitted on 27 Nov 2019

HAL is a multi-disciplinary open access archive for the deposit and dissemination of scientific research documents, whether they are published or not. The documents may come from teaching and research institutions in France or abroad, or from public or private research centers.
L'archive ouverte pluridisciplinaire HAL, est destinée au dépôt et à la diffusion de documents scientifiques de niveau recherche, publiés ou non, émanant des établissements d'enseignement et de recherche français ou étrangers, des laboratoires publics ou privés. 


\title{
NUMERICAL MODELLING OF TURBULENT TWO PHASE FLOWS USING THE TWO FLUID APPROACH
}

\author{
Jean-Marc Hérard ${ }^{1,2, *}$ \\ ${ }^{1}$ Université de Provence, Centre de Mathématiques et d'Informatique, \\ Laboratoire d'Analyse, Topologie et Probabilités - UMR CNRS 6632, \\ 39 rue Joliot Curie, 13453 Marseille cedex 13, FRANCE \\ herard@cmi.univ-mrs.fr \\ 2 Électricité de France, Division Recherche et Développement, \\ Département Mécanique des Fluides et Transferts Thermiques, \\ 6 quai Watier, 78401 Chatou cedex, FRANCE \\ herard@chi80bk.der.edf.fr
}

\begin{abstract}
This paper adresses the problem of turbulence modelling when focusing on two-fluid models with distinct pressures in each phase. This work represents the sequel of a previous contribution which was devoted to laminar flows. The turbulence effects are accounted for through a very simple model which only requires computing the so-called turbulent kinetic energy within each phase. Dissipation mechanism is represented by an algebraic relation. One shows that one may obtain a physically relevant entropy inequality while writing both interface velocity and interface pressure in terms of local unknowns. The whole convective set is a non strictly hyperbolic system, and involves three contact discontinuities, which require an accurate numerical treatment. Unlike in the pure laminar case, one cannot recast the whole convective set in a conservative form. This is simply due to the occurence of turbulent kinetic energies. Approximate jump conditions are introduced to close the true non conservative products arising in the governing equations of turbulent kinetic energies. Investigation of the field-by-field structure enables to establish that

\footnotetext{
* Senior Engineer in EDF, Associate Research Direc-
} tor at CNRS -UMR CNRS 6632-, Laboratoire d'Analyse Topologie et Probabilités, Université de Provence, AIAA member

Copyright (C) 2003 The American Institute of Aeronautics and astronautics, Inc. All rights reserved.
\end{abstract}

expected positive quantities should remain so. A few numerical experiments involving the standard two phase flow shock tube apparatus complete the whole.

\section{INTRODUCTION}

This paper focuses on the physical and numerical modelling of turbulent two phase flows, when one wishes to retain the two fluid approach without assuming local instantaneous presssure equilibrium. For a general presentation of the two fluid approach and the basics of ensemble averaging, the reader is refered to ${ }^{26},{ }^{3},{ }^{25},{ }^{10},{ }^{9},{ }^{39},{ }^{21}$ and $^{34}$ for instance. An overwhelming number of specific papers refers to the so-called single pressure two fluid model. This has motivated a large debate over the past twenty years, which mainly relies on the problem of the lack of hyperbolicity of the convective process in these sets of PDE. A partial review of potential problems and cures was recently described in $^{38}$ in the framework of the ASTAR project, and one may also refer to ${ }^{6}$ which discusses the problem of the maximum principle for the void fraction in this kind of models. Some ways to overcome the potential loss of hyperbolicity on coarse meshes have been suggested recently in ${ }^{7}$ and $^{28}$ among others. In the present approach, one no longer assumes the crude hypothesis of local pressure equilibrium between phases, and we follow the pioneering 
work of ${ }^{29},{ }^{1},{ }^{24},{ }^{27},{ }^{23}$ and other workers, though using different closures for the specific terms occuring, namely the interfacial pressure and the interfacial velocity.

This work is a sequel of a previous work described in ${ }^{15}$ and $^{8}$.

\section{$\underline{\text { GOVERNING EQUATIONS }}$}

The governing set of equations, which involves convective terms, source terms $S(W)$ (to account for the mass transfer, drag effects, energy exchange and dissipation mechanisms), may be written as (neglecting diffusive terms):

$\left\{\begin{array}{l}A(W) \frac{\partial W}{\partial t}+\frac{\partial F(W)}{\partial x}+C(W) \frac{\partial G(W)}{\partial x}=S(W) \\ W(x, 0)=W_{0}(x)\end{array}\right.$

with $W, F(W), G(W)$ and $S(W)$ in $\mathbb{R}^{9}$, and $C(W), A(W)$ in $\mathbb{R}^{9 \times 9}$. The variable $W$ is :

$$
W=\left(\begin{array}{c}
\alpha_{2} \\
\alpha_{2} \rho_{2} \\
\alpha_{2} \rho_{2} U_{2} \\
\alpha_{2} E_{2} \\
\alpha_{2} K_{2} \\
\alpha_{1} \rho_{1} \\
\alpha_{1} \rho_{1} U_{1} \\
\alpha_{1} E_{1} \\
\alpha_{1} K_{1}
\end{array}\right)
$$

All over the paper, $\alpha_{2}$ stands for the void fraction of phase referenced 2 , and $\alpha_{1}=1-\alpha_{2}$. The mean density, the mean velocity, the turbulent kinetic energy, and the total mean energy within phase $k$ are denoted $\rho_{k}, U_{k}, K_{k}$ and $E_{k}$ respectively. Viscous terms are included in the contribution $E(W)$. The conservative convective flux $F(W)$ reads :

$$
F(W)=\left(\begin{array}{c}
0 \\
\alpha_{2} \rho_{2} U_{2} \\
\alpha_{2}\left(\rho_{2} U_{2}^{2}+P_{2}+\frac{2 K_{2}}{3}\right) \\
\alpha_{2} U_{2}\left(E_{2}+P_{2}+\frac{2 K_{2}}{3}\right) \\
\alpha_{2} K_{2} U_{2} \\
\alpha_{1} \rho_{1} U_{1} \\
\alpha_{1}\left(\rho_{1} U_{1}^{2}+P_{1}+\frac{2 K_{1}}{3}\right) \\
\alpha_{1} U_{1}\left(E_{1}+P_{1}+\frac{2 K_{1}}{3}\right) \\
\alpha_{1} K_{1} U_{1}
\end{array}\right)
$$

$$
(A(W)-I) \frac{\partial W}{\partial t}=\left(\begin{array}{c}
0 \\
0 \\
0 \\
P_{i n t}^{2} \frac{\partial \alpha_{2}}{\partial t} \\
0 \\
0 \\
0 \\
P_{i n t}^{1} \frac{\partial \alpha_{1}}{\partial t} \\
0
\end{array}\right)
$$

and :

$$
C(W) \frac{\partial G(W)}{\partial x}=\left(\begin{array}{c}
V_{i} \frac{\partial \alpha_{2}}{\partial x} \\
0 \\
-P_{i n t}^{2} \frac{\partial \alpha_{2}}{\partial x} \\
0 \\
\frac{2 \alpha_{2} K_{2}}{3} \frac{\partial U_{2}}{\partial x} \\
0 \\
-P_{i n t}^{1} \frac{\partial \alpha_{1}}{\partial x} \\
0 \\
\frac{2 \alpha_{1} K_{1}}{3} \frac{\partial U_{1}}{\partial x}
\end{array}\right)
$$

The internal energy $e_{k}$ and the turbulent mean total energy $E_{k}$ are given through:

$$
\left\{\begin{array}{l}
e_{k}=e_{k}\left(P_{k}, \rho_{k}\right) \\
E_{k}=0.5 \rho_{k} U_{k} U_{k}+K_{k}+\rho_{k} e_{k}
\end{array}\right.
$$

First, we emphasize that the list of arguments for the mean internal energy imply rather crude assumptions on the statistical thermodynamics, unless one focuses on simple instantaneous EOS within each phase (such as perfect gas EOS or Tamman EOS for instance). Second, we also immediately assume the following closure law on interfacial pressures $P_{i n t}^{k}$ :

$$
P_{i n t}^{1}-P_{i n t}^{2}=0
$$

which enables to ensure the conservation principle of both the total momentum equation and total energy equation for the mixture. Extensions to the framework of situations where surface tensions cannot be neglected are not be discussed herein. The drag term, turbulent dissipation, and the pressure relaxation term require giving associated time scales $\tau_{\alpha}, \tau_{U}, \tau_{K_{k}}$ in: 


$$
S(W)=\left(\begin{array}{c}
-\alpha_{2}\left(1-\alpha_{2}\right) \frac{P_{1}-P_{2}}{\tau_{\alpha}\left(P_{1}+P_{2}\right)} \\
0 \\
\alpha_{2}\left(1-\alpha_{2}\right)\left(\rho_{1} \rho_{2}\right)^{1 / 2} \frac{U_{1}-U_{2}}{\tau_{U}} \\
V_{i} \alpha_{2}\left(1-\alpha_{2}\right)\left(\rho_{1} \rho_{2}\right)^{1 / 2} \frac{U_{1}-U_{2}}{\tau_{U}} \\
-\frac{\alpha_{2} K_{2}}{\tau_{K_{2}}} \\
0 \\
\alpha_{2}\left(1-\alpha_{2}\right)\left(\rho_{1} \rho_{2}\right)^{1 / 2} \frac{U_{2}-U_{1}}{\tau_{U}} \\
V_{i} \alpha_{2}\left(1-\alpha_{2}\right)\left(\rho_{1} \rho_{2}\right)^{1 / 2} \frac{U_{1}-U_{2}}{\tau_{U}} \\
-\frac{\alpha_{1} K_{1}}{\tau_{K_{1}}}
\end{array}\right)
$$

We choose data for $\tau_{K_{k}}$ in agreement with single phase turbulent literature (see for instance the work of Spalart ${ }^{37}$ or Baldwin-Lomax. ${ }^{2}$ ) Standard drag correlations $\left({ }^{21},{ }^{34},{ }^{35}\right)$ implicitely provide $\tau_{U}$, and may possibly account for interaction between the turbulent fields. The form of $\tau_{\alpha}$ is chosen in agreement with those used in the laminar framework $\left(\mathrm{see}^{31},{ }^{15},{ }^{8}\right)$. In order to detail both interfacial pressure and velocity fields $V_{i}$ and $P_{i}$, we from now set (these notations will be used throughout the paper):

$$
m_{k}=\alpha_{k} \rho_{k}
$$

and

$$
\begin{gathered}
\beta_{k}=\frac{m_{k}}{m_{1}+m_{2}} \\
\left\{\begin{array}{c}
P_{i}=\mu_{1}(W) P_{1}+\left(1-\mu_{1}(W)\right) P_{2} \\
+\mu_{2}(W)\left(m_{1} m_{2}\right)^{1 / 2}\left(U_{1}-U_{2}\right)^{2} \\
V_{i}=\beta(W) U_{1}+(1-\beta(W)) U_{2}
\end{array}\right.
\end{gathered}
$$

The basic principle which is involved is that closures should obey the local objective rule: $\left(U_{1}(x, t)=U_{2}(x, t)\right.$ and $\left.P_{1}(x, t)=P_{2}(x, t)\right) \mathrm{im}-$ ply: $V_{i}(x, t)=U_{1}(x, t)=U_{2}(x, t)$ and $P_{i}(x, t)=$ $P_{1}(x, t)=P_{2}(x, t)$. Scalar functions $\mu_{1}(W)(1-$ $\left.\left.\mu_{1}(W)\right), \mu_{2}(W)\right)$ and $\beta(W)(1-\beta(W))$ are assumed non negative. Following a previous work $\left({ }^{15},{ }^{8}\right)$, we will use the specific choice:

$$
\left\{\begin{array}{l}
\mu_{1}(W)=\frac{a_{1} \beta_{2}}{a_{1} \beta_{2}+a_{2} \beta_{1}} \\
\mu_{2}(W)=0 \\
\beta(W)=\beta_{1}
\end{array}\right.
$$

using the following definitions:

$$
a_{k}=\frac{1}{s_{k}} \frac{\frac{\partial s_{k}\left(P_{k}, \rho_{k}\right)}{\partial P_{k}}}{\frac{\partial e_{k}\left(P_{k}, \rho_{k}\right)}{\partial P_{k}}}
$$

where the specific entropy $s_{k}$ and the speed of density waves $c_{k}$ comply with:

$$
\left\{\begin{array}{c}
\left(c_{k}\right)^{2}=\frac{\frac{P_{k}}{\left(\rho_{k}\right)^{2}}-\frac{\partial e_{k}\left(P_{k}, \rho_{k}\right)}{\partial \rho_{k}}}{\frac{\partial e_{k}\left(P_{k}, \rho_{k}\right)}{\partial P_{k}}}=\frac{\hat{\gamma}_{k} P_{k}}{\rho_{k}} \\
\left(c_{k}\right)^{2} \frac{\partial s_{k}\left(P_{k}, \rho_{k}\right)}{\partial P_{k}}+\frac{\partial s_{k}\left(P_{k}, \rho_{k}\right)}{\partial \rho_{k}}=0
\end{array}\right.
$$

The reader is refered to the papers by Glimm and co authors ${ }^{22},{ }^{23}$ and also to the references ${ }^{24},{ }^{27}$. In the latter work in particular, the choice of the couple $P_{i}, V_{i}$ is precisely $P_{1}, U_{2}$ which is actually one particular example in the formulation above. In another recent work ${ }^{20}$, a similar formulation is used, setting: $V_{i}=U_{1}$ and : $P_{i}=P_{2}+\psi\left(\alpha_{2}\right)$. Some comments pertaining to the influence of the interface velocity will follow in a next section.

\section{MAIN PROPERTIES}

We provide below some of the main properties of the whole closed set of equations.

\section{Property 1.a}

The homogeneous convective subset :

$$
(I d+D(W)) \frac{\partial W}{\partial t}+\frac{\partial F(W)}{\partial x}+C(W) \frac{\partial G(W)}{\partial x}=0
$$

is hyperbolic, since it has real eigenvalues $\lambda_{k}$, and the associated right eigenvectors span the whole space $\mathbb{R}^{9}$-unless some resonance occurs-:

$$
\left\{\begin{array}{l}
\lambda_{1}=V_{i} \\
\lambda_{2}=U_{2}-\hat{c_{2}} \\
\lambda_{3,4}=U_{2} \\
\lambda_{5}=U_{2}+\hat{c_{2}} \\
\lambda_{6}=U_{1}-\hat{c_{1}} \\
\lambda_{7,8}=U_{1} \\
\lambda_{9}=U_{1}+\hat{c_{1}}
\end{array}\right.
$$

Problems arising with resonant waves are disregarded here, though of great importance ${ }^{13}$. The reader may have a look at ${ }^{33}$ and many references therein for a first approach. The exact expression of celerities shows the influence of the turbulent kinetic energy :

$$
\rho_{k}\left(\hat{c_{k}}\right)^{2}=\hat{\gamma}_{k} P_{k}+\frac{10 K_{k}}{9}
$$

which is the straightforward counterpart of what happens in single phase turbulent models. The physical significance of contribution proportional to $K_{k}$ may be easily understood by investigating 
the linearised system, and focusing on density perturbations, with given entropy rate $P_{k}\left(\rho_{k}\right)^{-\gamma_{k}}$ and turbulent entropy rate $K_{k}\left(\rho_{k}\right)^{-5 / 3}$ (assuming perfect gas EOS within each phase).

\section{Property $1 . b$}

Whatever the closure laws for $P_{i}$ and $V_{i}$ are, the $3,4,7,8$ fields are linearly degenerated, and the $2,5,6,9$ waves are genuinely non linear fields.

These results were expected owing to the form of the eigenstructure of the two-phase flow model in the laminar framework $\left({ }^{8}\right)$, and in the single phase turbulent frame $\left({ }^{11}\right)$. Note that these results do not depend on the specific choice of closure laws for $V_{i}$ and $P_{i}$. On the contrary, the following properties are tightly bound with the latter:

Property 2

Defining the entropy-entropy flux pair $\left(\eta, F_{\eta}\right)$ :

$$
\left\{\begin{array}{l}
\eta_{k}=\log \left(s_{k}\right)+\psi\left(\alpha_{2}\right) \\
\eta=-\alpha_{1} \rho_{1} \eta_{1}-\alpha_{2} \rho_{2} \eta_{2} \\
F_{\eta}=-\alpha_{1} \rho_{1} \eta_{1} U_{1}-\alpha_{2} \rho_{2} \eta_{2} U_{2}
\end{array}\right.
$$

the following entropy inequality holds for regular solutions of the above mentionned system :

$$
\frac{\partial \eta}{\partial t}+\frac{\partial F_{\eta}}{\partial x}<0
$$

This entropy inequality enables to select the admissible shock waves. The closure law for the interface velocity ensures that:

\section{Property 3}

We still set $\beta(W)=\beta_{1}$. The $1-$ field is linearly degenerated since it agrees with :

$$
\nabla_{W} V_{i}(W) \cdot r_{1}(W)=0
$$

where $r_{1}(W)$ stands for the right eigenvector associated with the first eigenvalue $\lambda_{1}(W)$.

Other possible choices correspond to the extreme values

$$
\beta(W)(1-\beta(W))=0 .
$$

In that case, it must emphasized that some specific behaviour arises, since the choice $V_{i}=U_{1}$ induces $P_{i}=P_{2}$, and thus the governing equations for variables $s_{1}, s_{2}$ become:

$$
\frac{\partial s_{k}}{\partial t}+U_{k} \frac{\partial s_{k}}{\partial x}=0 .
$$

An amazing point is that this clearly corresponds to one of the assumptions in the approach recently proposed by Gavrilyuk ( see $^{20}, 32$ ) This model is clearly no longer a mixture model but a true interface model. It also has some numerical consequences when using the rough Godunov schemes described in the next section. We also emphasize that an alternative way to obtain relevant closures for the interfacial pressure $P_{i}$ was proposed in ${ }^{8}$. Though it seems another interesting way to improve closure laws, this will not be discussed herein. We turn now to the description of the structure of waves.

\section{Property 4}

If $I_{k}$ refers to the list of Riemann invariants associated with the $k$-wave, one has the following result:

$$
\begin{aligned}
& I_{1}=V_{i}, \frac{m_{1} m_{2}}{m_{1}+m_{2}}\left(U_{1}-U_{2}\right), \frac{s_{2}}{s_{1}}, \hat{s_{1}}, \hat{s_{2}}, \\
& e_{1}+\frac{\pi_{1}+K_{1}}{\rho_{1}}+\frac{\left(U_{1}-V_{i}\right)^{2}}{2}, e_{2}+\frac{\pi_{2}+K_{2}}{\rho_{2}}+\frac{\left(U_{2}-V_{i}\right)^{2}}{2}, \\
& \frac{m_{1} m_{2}}{m_{1}+m_{2}}\left(U_{1}-U_{2}\right)^{2}+\alpha_{1} \pi_{1}+\alpha_{2} \pi_{2} \\
& I_{2}=\alpha_{2}, U_{2}+\psi_{2}, s_{2}, \hat{s_{2}}, \pi_{1}, U_{1}, s_{1}, \hat{s_{1}} \\
& I_{3-4}=\alpha_{2}, U_{2}, \pi_{2}, \pi_{1}, U_{1}, s_{1}, \hat{s_{1}} \\
& I_{5}=\alpha_{2}, U_{2}-\psi_{2}, s_{2}, \hat{s_{2}}, \pi_{1}, U_{1}, s_{1}, \hat{s_{1}} \\
& I_{6}=\alpha_{2}, U_{1}+\psi_{1}, s_{1}, \hat{s_{1}}, \pi_{2}, U_{2}, s_{2}, \hat{s_{2}} \\
& I_{7-8}=\alpha_{2}, U_{1}, \pi_{1}, \pi_{2}, U_{2}, s_{2}, \hat{s_{2}} \\
& I_{9}=\alpha_{2}, U_{1}-\psi_{1}, s_{1}, \hat{s_{1}}, \pi_{2}, U_{2}, s_{2}, \hat{s_{2}}
\end{aligned}
$$

noting :

$$
\begin{aligned}
& \psi_{k}=\int_{0}^{\rho_{k}}\left(\frac{\hat{c_{k}}\left(\rho_{k}, s_{k}, \hat{s_{k}}\right)}{\rho_{k}} d \rho_{k}\right) \\
& \pi_{k}=P_{k}+\frac{2 K_{k}}{3} \\
& \hat{s_{k}}=\frac{K_{k}}{\rho_{k}\left(m_{k}\right)^{2 / 3}}
\end{aligned}
$$

The Riemann invariants in the 1 - wave will be respectively noted $I_{1}^{k}$ afterwards for $k=1,8$. Obviously, inside the $k$-wave $(2 \leq k \leq 8)$, the explicit form of Riemann invariants ensures that all expected positive quantities remain positive in genuinely non linear fields, provided that no vacuum occurs in the solution in any phase. This result is equivalent of what happens in the laminar frame $\left({ }^{15}\right)$, for regular rarefaction waves. The specific case of the 1 wave will be discussed later on, since it is a Linearly Degenerate field.

Turning then to discontinuities, we denote as usual $[\phi]=\phi_{r}-\phi_{l}$, the jump between the two left and right states (labeled $l, r$ ) on each side of the discontinuity travelling at speed $\sigma$. We also define the mean $\bar{\phi}=\left(\phi_{r}+\phi_{l}\right) / 2$. For weak shocks, jump 
conditions may be written :

$$
\begin{aligned}
& \left.\left(\overline{V_{i}}-\sigma\right)\right) \Delta\left(\alpha_{k}\right)=0 \\
& \Delta\left(m_{k}\left(U_{k}-\sigma\right)\right)=0 \\
& \Delta\left(m_{k} U_{k}\left(U_{k}-\sigma\right)+\alpha_{k} \pi_{k}\right)-\hat{P}_{i} \Delta\left(\alpha_{k}\right)=0 \\
& \Delta\left(\alpha_{k} E_{k}\left(U_{k}-\sigma\right)+\alpha_{k} \pi_{k} U_{k}\right) \\
& -\hat{P}_{i} \overline{V_{i}} \Delta\left(\alpha_{k}\right)=0 \\
& \Delta\left(m_{k} \hat{s_{k}}\left(U_{k}-\sigma\right)\right)=0 .
\end{aligned}
$$

The latter are valid in the pure laminar frame $\left(K_{1}(x, t)=K_{2}(x, t)=0\right)$, and at least for weak shocks in turbulent single phase flows. On both sides of the 1-contact discontinuity, these make sense since $\Delta\left(\alpha_{k}\right)=0$, which results in the following jump relations within each phase:

$$
\begin{aligned}
& \Delta\left(\alpha_{k}\right)=0 \\
& \Delta\left(\rho_{k}\left(U_{k}-\sigma\right)\right)=0 \\
& \Delta\left(\rho_{k} U_{k}\left(U_{k}-\sigma\right)+\pi_{k}\right)=0 \\
& \Delta\left(E_{k}\left(U_{k}-\sigma\right)+\pi_{k} U_{k}\right)=0 \\
& \Delta\left(\rho_{k} \hat{s_{k}}\left(U_{k}-\sigma\right)\right)=0
\end{aligned}
$$

\section{Property 5}

Assume that the perfect gas EOS holds within each phase, that is: $P_{k}=\left(\gamma_{k}-1\right) \rho_{k} e_{k}$. Then above jump conditions ensure that through a shock wave, the right state is relevant, which means that:

$$
\left(\alpha_{k}\right)_{r}>0,\left(\rho_{k}\right)_{r}>0,\left(P_{k}\right)_{r}>0,\left(K_{k}\right)_{r}>0
$$

provided that no vacuum occurs in the solution and that the left state agrees with : $\left(\alpha_{k}\right)_{l}>0$, $\left(\rho_{k}\right)_{l}>0,\left(P_{k}\right)_{l}>0,\left(K_{k}\right)_{l}>0$. More over, the ratio of densities is bounded :

$$
\left(b_{k}\right)^{-1}<\frac{\left(\rho_{k}\right)_{r}}{\left(\rho_{k}\right)_{l}}<b_{k}
$$

and the rate of turbulence has a lower and an upper bound:

$$
1 \leq \sup \left(\frac{\left(K_{k}\right)_{r}}{\left(K_{k}\right)_{l}}, \frac{\left(K_{k}\right)_{l}}{\left(K_{k}\right)_{r}}\right) \leq\left(b_{k}\right)^{5 / 3}
$$

with $b_{k}=\frac{\gamma_{k}+1}{\gamma_{k}-1}$. The pressure ratio $\sup \left(\frac{\left(P_{k}\right)_{r}}{\left(P_{k}\right)_{l}}, \frac{\left(P_{k}\right)_{l}}{\left(P_{k}\right)_{r}}\right)$ has no upper bound.

We note that conditions on the ratio of the mean densities through a shock wave correspond to the conditions arising in the pure laminar framework (see for instance Smoller ${ }^{36}$ ). This is expected since the instantaneous inequality $a \phi_{l}<\phi_{r}<b \phi_{l}$ obviously provides the following relation between the mean quantities: $a<\phi_{l}>\leq<\phi_{r}>\leq b<\phi_{l}>$. We also recall that approximate jump conditions proposed in ${ }^{11}$ induce $1 / 4<\frac{\left(\rho_{k}\right)_{r}}{\left(\rho_{k}\right)_{l}}<4$, which is indeed more restrictive than the latter, since the usual condition $1<\gamma_{k}<5 / 3$ implies $4<b_{k}$. Moreover ${ }^{11}$ also implies that the ratio of turbulent kinetic energies is unbounded, unlike in the present case.

\section{Proof}

The proof is straightforward. Setting: $v_{k}=U_{k}-\sigma$, above jump conditions immediately provide the following relations between states through genuinely non linear simple fields associated with $\lambda_{2}$ or $\lambda_{6}$ :

$$
\begin{aligned}
& \left(\alpha_{k}\right)_{r}=\left(\alpha_{k}\right)_{l} \\
& \left(\rho_{k} v_{k}\right)_{r}=\left(\rho_{k} v_{k}\right)_{l} \\
& \left(\rho_{k}\right)_{r}=z_{k}\left(\rho_{k}\right)_{l} \\
& \left(U_{k}\right)_{r}=\left(U_{k}\right)_{l}-\left(\frac{\left(\pi_{k}\right)_{l}}{\left(\rho_{k}\right)_{l}}\left(\frac{\left(\pi_{k}\right)_{r}}{\left(\pi_{k}\right)_{l}}-1\right)\left(\frac{z_{k}-1}{z_{k}}\right)\right)^{1 / 2} \\
& \left(K_{k}\right)_{r}=\left(z_{k}\right)^{5 / 3}\left(K_{k}\right)_{l} \\
& \left(P_{k}\right)_{r}=\left(P_{k}\right)_{l} \frac{b_{k} z_{k}-1}{b_{k}-z_{k}} \\
& +\frac{2\left(K_{k}\right)_{l}}{3\left(b_{k}-z_{k}\right)}\left(-4\left(\left(z_{k}\right)^{5 / 3}-z_{k}\right)+\left(\left(z_{k}\right)^{8 / 3}-1\right)\right)
\end{aligned}
$$

with $z_{k}>1$. The connection of both the pressure and the turbulent kinetic energy on each side of the shock is slightly distinct from the one introduced $\mathrm{in}^{11}$, at least for shocks which are not too weak. Nonetheless, the true non-conservative products theoretically inhibit investigating such a situation. A similar proof holds through simple waves connected with eigenvalues $\lambda_{5}$ or $\lambda_{9}$ (with $\left.z_{k}<1\right)$.

When focusing on simple EOS such as those considered in our work (perefect gas EOS or alternatively Tamman EOS within each phase), these approximate jump conditions also preserve the positivity constraints through the 1 -wave:

\section{Property 6}

We still assume the form of $V_{i}$ with $\beta(W)=\beta_{1}$. We also still restrict to the framework :

$$
P_{k}=\left(\gamma_{k}-1\right) \rho_{k} e_{k}
$$

with $1<\gamma_{k}<5 / 3$. Then, the connection of states through the 1 -wave ensures that $\alpha_{k}>0$ but also:

$$
m_{k}>0, P_{k}>0, K_{k}>0
$$

\section{Proof}

Obviously, the only wave through which the void fraction varies is the $1-$ wave, thus: $\alpha_{l}=\alpha_{L}$ and also: $\alpha_{r}=\alpha_{R}$. Through the $1-$ contact disconti- 
nuity, jump conditions yield:

$$
\begin{aligned}
& \sigma=\overline{V_{i}}=\left(V_{i}\right)_{l}=\left(V_{i}\right)_{r} \\
& \Delta\left(m_{k}\left(U_{k}-\sigma\right)\right)=0 \\
& \Delta\left(m_{k} \hat{s_{k}}\left(U_{k}-\sigma\right)\right)=0 \\
& \Delta\left(\alpha_{k} E_{k}\left(U_{k}-\sigma\right)+\alpha_{k} \pi_{k} U_{k}\right) \\
& -\overline{V_{i}} \Delta\left(m_{k} U_{k}\left(U_{k}-\sigma\right)+\alpha_{k} \pi_{k}\right)=0 \\
& \Delta\left(m_{1} U_{1}\left(U_{1}-\sigma\right)+\alpha_{1} \pi_{1}\right) \\
& +\Delta\left(m_{2} U_{2}\left(U_{2}-\sigma\right)+\alpha_{2} \pi_{2}\right)=0 \\
& \Delta\left(\frac{s_{2}}{s_{1}}\right)=0
\end{aligned}
$$

We still denote with subscript $l$ quantities on the left side on the 1 - wave. We recall that connecting states through this wave with help of Riemann invariants or jump relations is perfectly identical and leads to:

$$
\left(\gamma_{k} e_{k}+\frac{5\left(\hat{s_{k}}\right)_{l} m_{k}^{2 / 3}}{3}+\frac{Q_{l}^{2}}{2 m_{k}^{2}}\right)_{r}=\left(C_{k}\right)_{l}
$$

setting :

$$
\begin{aligned}
& Q_{l}=I_{1}^{2}\left(W_{l}\right)=\left(\frac{m_{1} m_{2}}{m_{1}+m_{2}}\left(U_{1}-U_{2}\right)\right)_{l} \\
& \left(C_{1}\right)_{l}=I_{1}^{6}\left(W_{l}\right)=\left(e_{1}+\frac{\pi_{1}+K_{1}}{\rho_{1}}+\frac{\left(U_{1}-V_{i}\right)^{2}}{2}\right)_{l} \\
& \left(C_{2}\right)_{l}=I_{1}^{7}\left(W_{l}\right)=\left(e_{2}+\frac{\pi_{2}+K_{2}}{\rho_{2}}+\frac{\left(U_{2}-V_{i}\right)^{2}}{2}\right)_{l} \\
& E_{l}=I_{1}^{8}\left(W_{l}\right)
\end{aligned}
$$

The main two equations correspond to the conservation of the total energy for both phases:

$$
\begin{aligned}
& Q_{l}^{2}\left(\frac{\gamma_{1}+1}{2 \gamma_{1} m_{1}}+\frac{\gamma_{2}+1}{2 \gamma_{2} m_{2}}\right)_{r} \\
& +\left(\frac{\gamma_{1}-1}{\gamma_{1}}\left(C_{1}\right)_{l} m_{1}+\frac{\gamma_{2}-1}{\gamma_{2}}\left(C_{2}\right)_{l} m_{2}\right)_{r} \\
& +\left(\frac{5-3 \gamma_{1}}{3 \gamma_{1}}\left(\hat{s_{1}}\right)_{l}\left(m_{1}\right)^{5 / 3}\right. \\
& \left.+\frac{5-3 \gamma_{2}}{3 \gamma_{2}}\left(\hat{s_{2}}\right)_{l}\left(m_{2}\right)^{5 / 3}\right)_{r}=E_{l}
\end{aligned}
$$

and the constant ratio of phase entropies:

$$
\begin{aligned}
& \left(\alpha_{1}\right)_{r}^{\gamma_{1}-1} \frac{\gamma_{1}-1}{\gamma_{1}}\left(m_{1}\right)_{r}^{1-\gamma_{1}} \\
& \left(\left(C_{1}\right)_{l}-\frac{Q_{l}^{2}}{2\left(m_{1}\right)_{r}^{2}}-\frac{5}{3}\left(\hat{s_{1}}\right)_{l}\left(m_{1}\right)_{r}^{2 / 3}\right) \\
& =\left(\frac{s_{2}}{s_{1}}\right)_{l}\left(\alpha_{2}\right)_{r}^{\gamma_{2}}-1 \frac{\gamma_{2}-1}{\gamma_{2}}\left(m_{2}\right)_{r}^{1-\gamma_{2}} \\
& \left(\left(C_{2}\right)_{l}-\frac{Q_{l}^{2}}{2\left(m_{2}\right)_{r}^{2}}-\frac{5}{3}\left(\hat{s_{2}}\right)_{l}\left(m_{2}\right)_{r}^{2 / 3}\right)
\end{aligned}
$$

This provides a coupled set of two non-linear equations with two unknowns $\left(m_{1}\right)_{r}$ and $\left(m_{2}\right)_{r}$. Performing implicit elimination of $\left(m_{1}\right)_{r}$ through the last equation and introducing in the former enables to compute solution in terms of only one unknown $\left(m_{2}\right)_{r}$. One may then check that the resulting equation has either zero, one or two positive solutions. As a consequence, the partial mass $\left(m_{1}\right)_{r}$ is also positive. Though not totally straightforward, a similar remark holds for internal energies $e_{k}$ and obviously for turbulent kinetic energies $K_{k}$, owing to the invariance of turbulent kinetic entropies through the one wave: $\hat{s_{k r}}=\hat{s_{k l}}$.

On the whole, it clearly appears that there is no major difference arising in the turbulent framework, when compared with the laminar ap$\operatorname{proach}^{8},{ }^{15}$.

\section{NumeriCAL METHOD}

We only provide here the main guidelines of the numerical method and a few numerical results. Actually, we use the same fractional step approach as in ${ }^{15}$ to compute the whole set. Thus we first compute an approximate solution of the homogeneous hyperbolic system :

$$
\left\{\begin{array}{l}
\frac{\partial W}{\partial t}+\frac{\partial F(W)}{\partial x}+C^{\prime}(W) \frac{\partial G(W)}{\partial x}=0 \\
W(x, 0)=W_{n}(x)
\end{array}\right.
$$

over $[t, t+\delta t]$, with given adequate boundary conditions. We then look for solutions of the ODE:

$$
\left\{\begin{array}{l}
\frac{\partial W}{\partial t}=S(W) \\
W(x, 0)=W^{*}(x)
\end{array}\right.
$$

provided initial data $W^{*}(x)$ given by an approximation of the solution of the first step at time $t+\delta t$. The second step is once again split in two, so that one first computes the ODE:

$$
\frac{\partial W}{\partial t}=S_{1}(W)=\left(\begin{array}{c}
0 \\
0 \\
\frac{\alpha_{1} \alpha_{2}\left(\rho_{1} \rho_{2}\right)^{1 / 2}}{\tau_{U}}\left(U_{1}-U_{2}\right) \\
\frac{\alpha_{1} \alpha_{2}\left(\rho_{1} \rho_{2}\right)^{1 / 2}}{\tau_{U}} V_{i}\left(U_{1}-U_{2}\right) \\
-\frac{\alpha_{2} K_{2}}{\tau_{K}} \\
0 \\
-\frac{\alpha_{1} \alpha_{2}\left(\rho_{1} \rho_{2}\right)^{1 / 2}}{\tau_{U}}\left(U_{1}-U_{2}\right) \\
-\frac{\alpha_{1} \alpha_{2}\left(\rho_{1} \rho_{2}\right)^{1 / 2}}{\tau_{U}} V_{i}\left(U_{1}-U_{2}\right) \\
-\frac{\alpha_{1} K_{1}}{\tau_{K_{1}}}
\end{array}\right)
$$

Through the latter the following behaviour is ensured:

$$
\begin{gathered}
m_{1} \frac{\partial e_{1}}{\partial P_{1}} \frac{\partial P_{1}}{\partial t}=\frac{M}{\tau_{U}}\left(1-\beta_{1}\right)\left(U_{1}-U_{2}\right)^{2}+\frac{\alpha_{1} K_{1}}{\tau_{K_{1}}} \\
m_{2} \frac{\partial e_{2}}{\partial P_{2}} \frac{\partial P_{2}}{\partial t}=\frac{M}{\tau_{U}} \beta_{1}\left(U_{1}-U_{2}\right)^{2}+\frac{\alpha_{2} K_{2}}{\tau_{K_{2}}} \\
M=\alpha_{1} \alpha_{2}\left(\rho_{1} \rho_{2}\right)^{1 / 2}
\end{gathered}
$$

Hence, as soon as $0 \leq \frac{\partial e_{k}}{\partial P_{k}}$, both pressures are increasing, which guarantees positive values of $P_{1}$ and $P_{2}$ during this step. Moreover, velocities follow the rule $\frac{\partial\left(U_{1}-U_{2}\right)^{2}}{\partial t} \leq 0$. Since 
$\frac{\partial \alpha_{2}}{\partial t}=\frac{\partial m_{k}}{\partial t}=0$, the void fraction and the densities $\rho_{k}$ remain constant wrt time.

Then, one needs to update values of pressure, density and void fractions through:

$$
\frac{\partial W}{\partial t}=S(W)-S_{1}(W)
$$

(obviously here, both $\frac{\partial U_{k}}{\partial t}$ and $\frac{\partial K_{k}}{\partial t}$ are null). In this latter step, both pressures relax towards an equilibrium state and remain positive meanwhile. Even more, the void fraction remains in its bounds, if: $0 \leq \hat{\gamma}_{k}+\left(P_{i}-P_{k}\right)\left(\rho_{k} P_{k} \frac{\partial e_{k}}{\partial P_{k}}\right)^{-1}$ (the proof is exactly the same as the one given $\mathrm{in}^{15}$ ). Actually, the computation of the second step involving ODE is not that obvious, and the reader is refered to ${ }^{15}$ for details. As it was expected, but surprisingly not clearly explained in the literature, this second step indeed ensures the relaxation of pressures $P_{1}$ and $P_{2}$ towards an equilibrium state.

On the whole, the first, second and third step preserve the maximum principle for the void fraction. More over, for finite time interval $[0, T]$, the turbulent kinetic energy remains positive within each phase, assuming of course positive inlet boundary conditions, positive initial conditions, and also assuming that $U_{k}, \frac{\partial U_{k}}{\partial x}$ and $\frac{1}{\tau_{K_{k}}}$ remain bounded. This result is only valid for regular solutions.

Approximate solutions of the latter first-order homogeneous system may be obtained applying for an extension of Rusanov scheme ${ }^{30}$, or the approximate Godunov scheme called VFRoencv. Details on the latter, which is based on the early proposal $^{19}$, can be found for instance in ${ }^{5}, 4$. More details on the speed of convergence of this family of schemes may be found in ${ }^{16}$ and $^{14}$. Extensions of these schemes to the frame of mixed type equations is also described in ${ }^{18}$, which is based on the pioneering work of A.Y. LeRoux and co workers. An important point to note is that an equivalent form of the latter homogeneous system may be written (the explicit form of the matrix $\mathrm{C}(\mathrm{Z})$ is given in appendix $\mathrm{A}$ ):

$$
\frac{\partial Z}{\partial t}+C(Z) \frac{\partial Z}{\partial x}=0
$$

when restricting to regular solutions, and noting:

$$
Z=\left(\alpha_{2}, s_{2}, \hat{s}_{2}, U_{2}, \pi_{2}, s_{1}, \hat{s}_{1}, U_{1}, \pi_{1}\right)
$$

$$
\begin{gathered}
A_{k}=\frac{\partial s_{k}}{\partial P_{k}}\left(P_{i}-P_{k}\right)\left(m_{k} \frac{\partial e_{k}}{\partial P_{k}}\right)^{-1} \\
B_{k}=\frac{2 K_{k}}{3 \alpha_{k}}+\frac{\hat{\gamma_{k}} P_{k}}{\alpha_{k}}+\left(P_{i}-P_{k}\right)\left(m_{k} \frac{\partial e_{k}}{\partial P_{k}}\right)^{-1}
\end{gathered}
$$

It may prevent the occurence of negative interface values of expected discrete quantities, with reasonable CFL constraint on the time stepping, in single phase areas ${ }^{17}$. This enables to develop a rather efficient solver, following the ideas developed in ${ }^{15}$. Eventually, it is emphasized that we compute here approximate solutions of the non-conservative equation of $K_{k}$.

The first series of figures (Fig. 1-4) displays the behaviour of the approximate solutions obtained when computing an unsteady shock tube in the turbulent framework, using perfect gas EOS within each phase $\left(\gamma_{1}=1.4, \gamma_{2}=1.2\right)$. The mesh contains 10000 regular cells. The CFL number is set to $1 / 2$. The initial data is : $\left(U_{1}\right)_{L}=\left(U_{2}\right)_{L}=0,\left(P_{1}\right)_{L}=\left(P_{2}\right)_{L}=10^{5}$, $\left(K_{1}\right)_{L}=\left(K_{2}\right)_{L}=10^{3},\left(\rho_{1}\right)_{L}=\left(\rho_{2}\right)_{L}=1$, $\left(\alpha_{1}\right)_{L}=0.6$, and : $\left(U_{1}\right)_{R}=\left(U_{2}\right)_{R}=0$, $\left(P_{1}\right)_{R}=\left(P_{2}\right)_{R}=10^{4},\left(K_{1}\right)_{R}=\left(K_{2}\right)_{R}=10^{2}$, $\left(\rho_{1}\right)_{R}=\left(\rho_{2}\right)_{R}=0.125,\left(\alpha_{1}\right)_{R}=0.5$, where subscripts $L$ and $R$ refer to $x<0.5$ and $x>0.5$ repectively. The pressure $P_{k}$ varies through the three contact discontinuities associated with $U_{1}$, $U_{2}$ and $V_{i}$, which are close one to another in this example. The turbulent kinetic energies also vary through the latter. However, $\pi_{k}$ remains constant through both fields associated with: $\lambda=U_{1}$ and $\lambda=U_{2}$. The void fraction, which is governed by a LD field, converges very slowly (as $h^{1 / 2}$ ), and this renders accurate computations rather uneasy. Extension to so-called 'second-order' is thus mandatory. The accuracy may be expected even worse on $2 \mathrm{D}$ or $3 \mathrm{D}$ meshes.

The last figures (Fig. 5 to 7 ) compare results obtained with the same initial condition, when using $V_{i}=\beta_{1} U_{1}+\left(1-\beta_{1}\right) U_{2}$ and $V_{i}^{\prime}=U_{2}$ alternatively.

\section{Conclusion}

As happens when computing laminar flows with help of the two fluid two pressure model, fine meshes are required in order to get a sufficiently accurate approximation of solutions of the whole set of PDE. One of the major reasons is that three distinct contact discontinuities are involved herein - which in addition are close to one another-, and this implies that very high order 
schemes or alternatively very fine meshes with low order schemes are used for preditions. This has motivated the building of new schemes ${ }^{14},{ }^{12}$ (that is mixed conservative - non conservative schemes) since conservative upwind sechemes only ensure a small rate of convergence $\left(h^{\frac{p}{p+1}}\right)$ for expected schemes of order $p$, when LD fields are present in the eigenstruture. This represents a great challenge for future work.

The author has benefited from financial support of the CEA-EDF project NEPTUNE

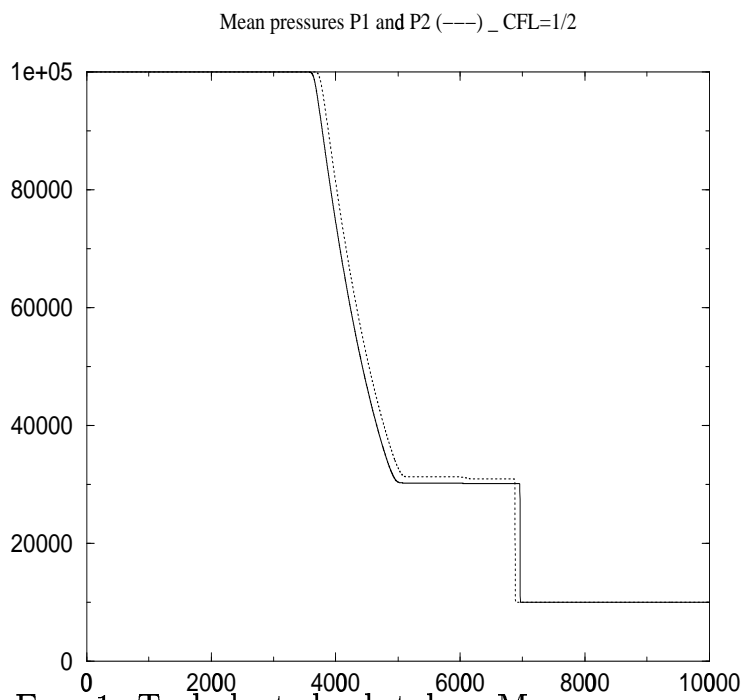

FIG. 1: Turbulent shock tube - Mean pressures

Turbulent kinetic energies $\mathrm{K} 1$ and $\mathrm{K} 2(---) \_\mathrm{CFL}=1 / 2$

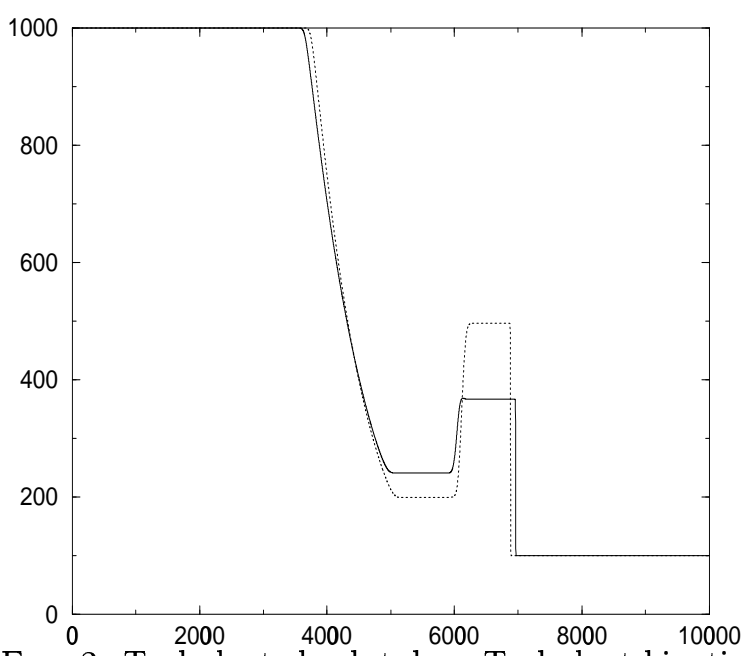

FIG. 2: Turbulent shock tube - Turbulent kinetic energies

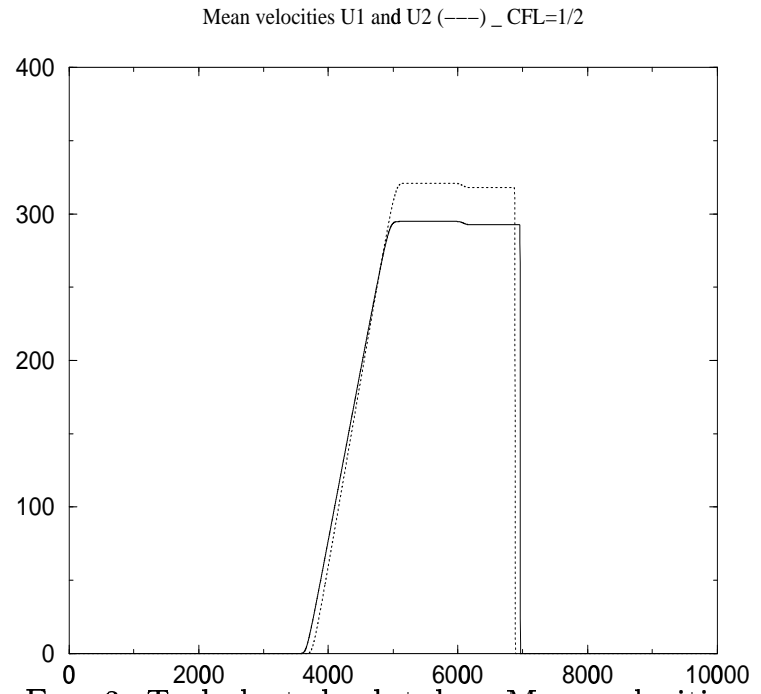

FIG. 3: Turbulent shock tube - Mean velocities
Mean densities rho_1 and rho_2 (---) and void fraction _ CFL=1/2

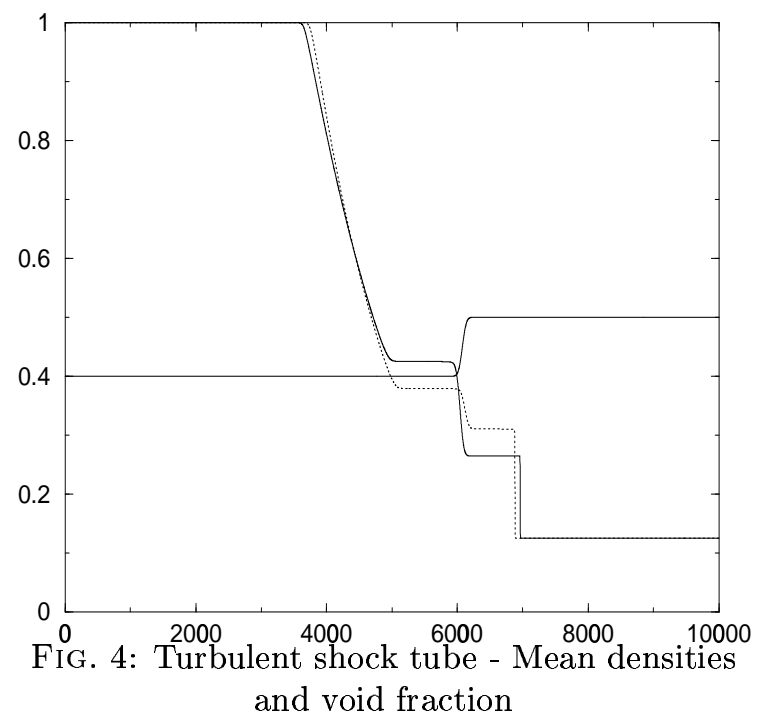

American Institute of Aeronautics and Astronautics 


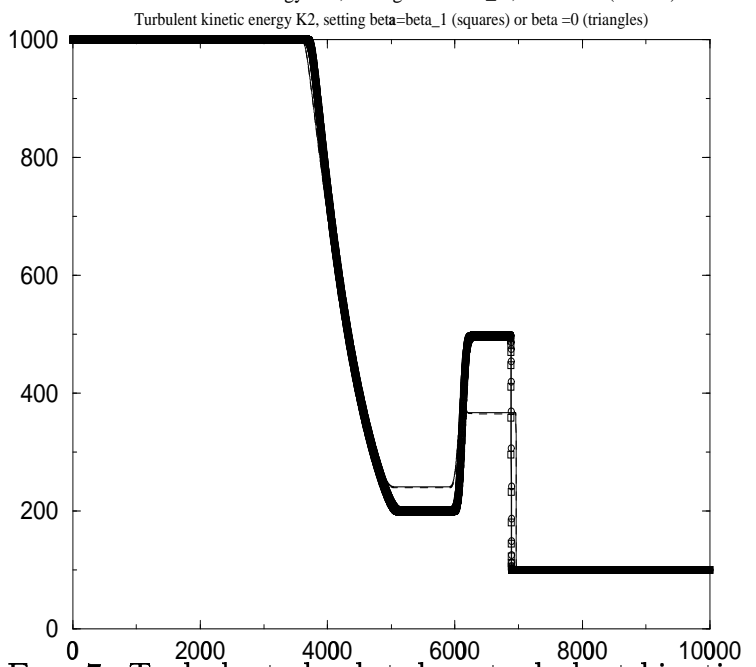

FIG. 7: Turbulent shock tube - turbulent kinetic energies for two distinct values of $V_{i}$

\section{REFERENCES}

[1] M.R. Baer And J.W. Nunziato, A two phase mixture theory for the deflagration to detonation (ddt) transition in reactive granular materials, Int. J. for Multiphase Flow, 1986, vol. 12-6, pp. 861-889.

[2] B.S. Baldwin and H. Lomax, Thin layer approximation and algebraic model for seperated turbulent flows, AIAA paper 78-25\%, 1978.

Mean velocity U1, setting beta $=$ beta $\_1$, or beta $=0$ (dashed $)$

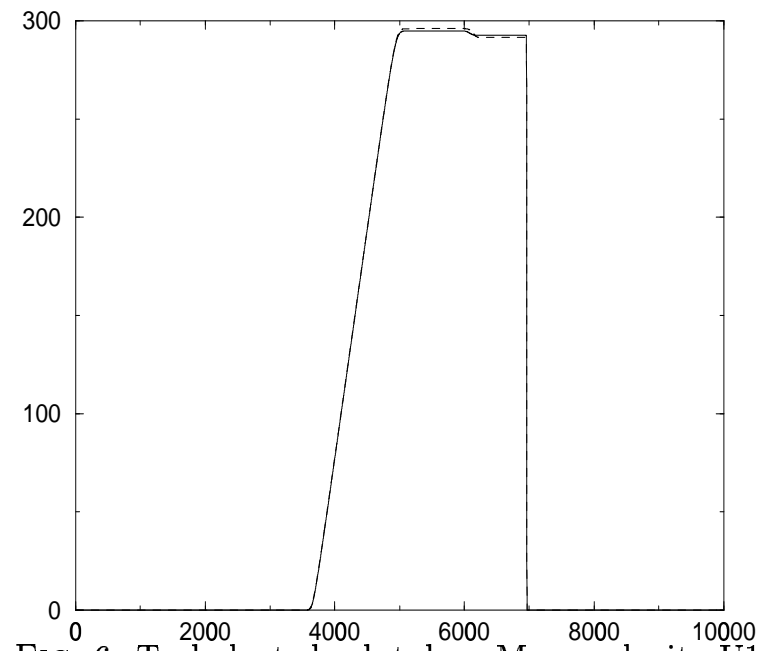

FIG. 6: Turbulent shock tube - Mean velocity U1 for two distinct values of $V_{i}$

[3] J.A. Boure, On a unified presentation of the non-equilibrium two phase flow models in non-equilibrium two phase flows, Proc. of the ASME Symposium- New York, 1975.

[4] T. Buffard, T. Gallouët and J.M. HÉRARD, A naive Godunov scheme to compute a non-conservative hyperbolic system, Int. Series Num. Math., 1998, vol. 129, pp. $129-138$.

[5] — A sequel to a rough Godunov scheme. Application to real gas flows, Computers and Fluids, 2000, vol. 29-7, pp. 813-847.

[6] S. Clerc, T. Gallouët, J.M. Hérard And N. SEguin, Partial review of positivity constraints in some two phase flow models, AIA A paper 2002-3185, 2002.

[7] F. Coquel, K. El Amine, E. Godlewski, B. Perthame et al., A numerical method using upwind schemes for the resolution of two 
phase flows, J. Comp. Phys., 1997, vol. 136, pp. $272-288$.

[8] F. Coquel, T. Gallouët, J.M. Hérard AND N. SEguin, Closure laws for a two-fluid two-pressure model, C. R. Acad. Sci. Paris, 2002, vol. I-334, pp. 927-932.

[9] D.A. Drew, Mathematical modelling of two phase flows, Annual Review of Fluid Mechanics, 1983, vol. 15, pp. 261-291.

[10] D.A. Drew and R.T. Lahey JR, Application of general constitutive principle to the derivation of multidimensional two phase flow equations, Int. J. for Multiphase Flow, 1979, vol. 5 , pp. $243-264$.

[11] A. Forestier, J.M. HÉrard AND $\mathrm{X}$. Louis, A Godunov type solver to compute turbulent compressible flows, C. R. Acad. Sci. Paris, 1997, vol. I-324, pp. 919-926.

[12] T. Gallouët, J.M. Hérard, R. Herbin, N. Jullian et al., Upwind finite volume schemes for one and two dimensional euler equations, AIAA paper 2003-4122, 2003.

[13] T. GallouËt, J.M. HÉrard AND N. Seguin, Approximation by finite volume schemes of a two fluid two pressure model for two phase flows, Proceedings of FVCA3, Hermes Penton Sciences, R. Herbin and D. Kroner editors, 2002.

[14] - A hybrid scheme to compute contact discontinuities in one dimensional euler systems, Math. Mod. and Num. Analysis, 2002, no36, pp. 1133-1159.

[15] — , Numerical modelling of two-phase flows using the two-fluid two-pressure approach, to appear in Math. Mod. Meth. in Appl. Sci., also in HI-81/01/043/A, 2002.

[16] _ Some recent Finite Volume schemes to compute Euler equations using real gas EOS, Int. J. for Num. Meth. in Fluids, 2002, vol. 39 , pp. 1073-1138.

[17] - On the use of some symmetrizing variables to deal with vacuum, to appear in Calcolo, 2003.

[18] — Some approximate Godunov schemes to compute shallow water equations with topography, Computers and Fluids, 2003, vol. 32 , pp. $479-513$.
[19] T. GallouËt and J.M. Masella, A rough Godunov scheme, C. R. Acad. Sci. Paris, 1996, vol. I-323, pp. 77-84.

[20] S. Gavrilyuk and R. Saurel, Mathematical and numerical modelling of two phase compressible flows with inertia, J. Comp. Phys., 2002, vol. 175, pp. 326-360.

[21] D. Gidaspow, Multiphase flow and fluidization, Academic Press, 1993.

[22] J. Glimm, D. Saltz and D.H. Sharp, Renormalization group solution of two phase flow equations for rayleigh-taylor mixing, Phys. Lett. A, 1996, vol. 222, pp. 171-176.

[23] — Two phase flow modelling of a fluid mixing layer, Journal of Fluid Mechanics, 1999, vol. 378, pp. 119-143.

[24] K.A. Gonthier And J.M. Powers, A high resolution method for a two phase model of deflagration to detonation transition, $J$. Comp. Phys., 2000, vol. 163, pp. 376-433.

[25] W.G. Gray and P.C.Y. Lee, On the theorems for local volume averaging of multiphase systems, Int. J. for Multiphase Flow, 1977, vol. I-3, pp. 333-340.

[26] M. IsHII, Thermo-fluid dynamic theory of two-phase flows, Collection de la Direction des Etudes et Recherches d'Electicité de France, 1975.

[27] A.K. Kapila, S.F. Son, J.B. Bdzil, R. Menikoff et al., Two-phase modelling of ddt : structure of the velocity relaxation zone, Physics of Fluids, 1997, vol. 9, pp. 3885-3897.

[28] H. Paillere, C. Corre and J.R. CasCALES, On the extension of the AUSM+ scheme to compressible two fluid models, Computers and Fluids, 2003, vol. 32, pp. 891916.

[29] V. Ransom and D.L. Hicks, Hyperbolic two-pressure models for two-phase flow, $J$. Comp. Phys., 1984, vol. 53, pp. 124-151.

[30] V.V. Rusanov, Calculation of interaction of non-steady shock waves with obstacles, J. Comp. Math. Phys. USSR, 1961, vol. 1, pp. 267-279.

\section{0}

American Institute of Aeronautics and Astronautics 
[31] R. Saurel and R. Abgrall, A multiphase godunov method for compressible multifluid and multiphase flows, J. Comp. Phys., 1999, vol. 150 , pp. $425-467$.

[32] R. Saurel, S. Gavrilyuk and F. Renaud, A multiphase model with internal degrees of freedom: application to shock bubble interaction, Journal of Fluid Mechanics, to appear.

[33] N. Seguin And J. Vovelle, Analysis and approximation of a scalar conservation law with a flux function with discontinuous coefficients, to appear in Math. Mod. and Methods in Applied Sciences, 2002.

[34] O. Simonin, Modélisation numérique des écoulements turbulents diphasiques à inclusions dispersées, Ecole de printemps de mécanique des fluides numérique, 1995.

[35] — Continuum modelling of dispersed turbulent two phase flows, Von Karmann Institute Lecture Series 96, 1996.

[36] J. SMOLLER, Shock waves and reaction diffusion equations, Springer Verlag, 1983.

[37] P. Spalart and S. Allmaras, A one equation turbulence model for aerodynamic flows, AIAA paper 92-0439, 1992.

[38] H. Staedtke, Remarks on single pressure two-fluid model and hyperbolicity, contribution to the ASTAR project, unpublished, august 2002 .

[39] H.B. Stewart and B. Wendroff, Twophase flow : models and methods, J. Comp. Phys., 1984, vol. 56, pp. 363-409.

\section{Appendix A}

We give below the form of the matrix of convective terms.

$$
C(Z)=\left(\begin{array}{ccccccccc}
V_{i} & 0 & 0 & 0 & 0 & 0 & 0 & 0 & 0 \\
A_{2}\left(U_{2}-V_{i}\right) & U_{2} & 0 & 0 & 0 & 0 & 0 & 0 & 0 \\
0 & 0 & U_{2} & 0 & 0 & 0 & 0 & 0 & 0 \\
\left(\pi_{2}-P_{i}\right) / m_{2} & 0 & 0 & U_{2} & \tau_{2} & 0 & 0 & 0 & 0 \\
B_{2}\left(U_{2}-V_{i}\right) & 0 & 0 & \gamma_{2} P_{2}+10 K_{2} / 9 & U_{2} & 0 & 0 & 0 & 0 \\
-A_{1}\left(U_{1}-V_{i}\right) & 0 & 0 & 0 & 0 & U_{1} & 0 & 0 & 0 \\
0 & 0 & 0 & 0 & 0 & 0 & U_{1} & 0 & 0 \\
-\left(\pi_{1}-P_{i}\right) / m_{1} & 0 & 0 & 0 & 0 & 0 & 0 & U_{1} & \tau_{1} \\
-B_{1}\left(U_{1}-V_{i}\right) & 0 & 0 & 0 & 0 & 0 & 0 & \gamma_{1} P_{1}+10 K_{1} / 9 & U_{1}
\end{array}\right)
$$

